\title{
Maxillary Sinus Lift Up: An Indirect Approach for Implant Placement in Posterior Maxilla
}

Amit Agnihotri, Deepshikha Agnihotri

\begin{abstract}
The posterior maxilla presents several challenges to the implantologist. Implant dentistry has become an excellent treatment modality since its inception into the modern era of dentistry. When patients present with deficient alveolar ridges, it could jeopardize the application of implant dentistry. This problem is especially magnified in the posterior maxilla where ridge resorption and sinus pneumatization are often encountered. The procedure of choice to restore this anatomic deficiency is maxillary sinus floor lift up. The purpose of this case report is to bring forward such technique which prevents perforation of sinus lining during implant placement by doing the sinus lifting with indirect approach through alveolar crest. The indirect approach for sinus lift is less invasive and less complex and therefore a better alternative for such cases.
\end{abstract}

Keywords: Maxillary sinus floor lift up, Implant, Indirect approach, Posterior maxilla.

How to cite this article: Agnihotri A, Agnihotri D. Maxillary Sinus Lift Up: An Indirect Approach for Implant Placement in Posterior Maxilla. Int J Oral Implant Clin Res 2012;3(2):101-104.

Source of support: Nil

Conflict of interest: None declared

\section{INTRODUCTION}

An adequate quantity and quality of bone is essential for successful implant therapy. The posterior edentulous maxilla presents special challenges to the implantologist that is unique to this region compared with the other areas of the mouth. Most important among these is the presence of the maxillary sinus. The Schneiderian membrane, which lines the sinus, is adherent to the underlying bone. The structures beneath the sinus consist of the alveolar ridge and the maxillary posterior teeth. ${ }^{1}$ As the edentulous area continues to atrophy, there is a continuing loss of bone height and density and an increase in antral pneumatization. ${ }^{2,3}$ It is therefore common to find the sinus floor close to the alveolar crest. This finding is related to two phenomena: (1) The enlargement of the sinus at the expense of the alveolus after tooth extraction because of the increased osteoclastic activity of the periosteum of the Schneiderian membrane ${ }^{4}$ and (2) increased pneumatization of the sinus because of the increase in positive intra-antral pressure. ${ }^{5}$ The posterior maxilla often presents specific problems for the placement of dental implants. The poor bone quality encountered in this region, in conjunction with inadequate bone volume is related to both, the size of the maxillary sinus and the resorption of the alveolar ridge.
The most widely used approaches for sinus lifting are: (1) The direct approach which includes approaching the sinus laterally using either one step or two step antrostomy, (2) the indirect approach which includes approaching the sinus through alveolar crest using an osteotome. The indirect approach being less invasive is more preferred over the direct one. This case report describes one such indirect approach for maxillary sinus lift up in which implant placement was carried out simultaneously with elevation of the sinus floor.

\section{CASE REPORT}

A 30-year-old male patient reported with the chief complaint of difficulty in chewing from right side because of loss of tooth in right upper back tooth region. On oral examination, right first molar was missing (Figs $1 \mathrm{~A}$ and $\mathrm{B}$ ). On radiographic examination, the available bone height in molar
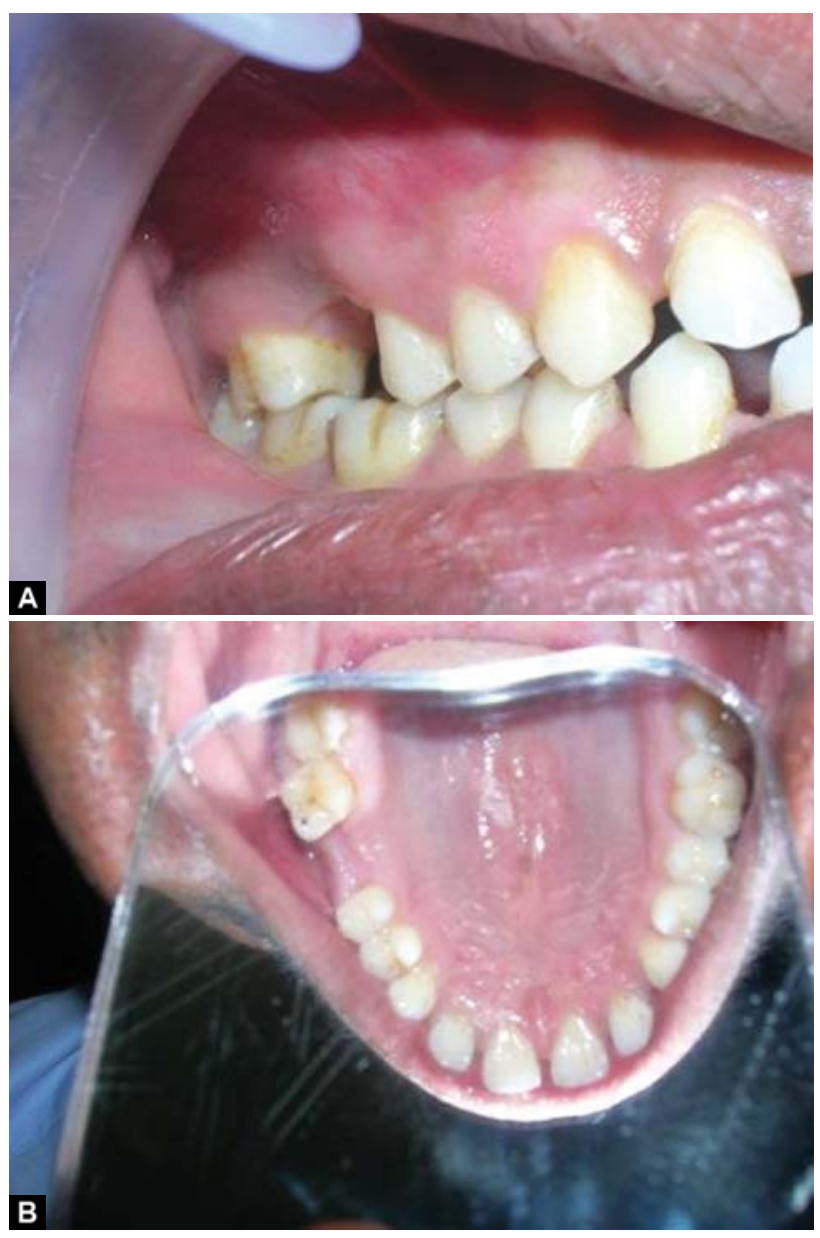

Figs 1A and B: Preoperative photographs showing site for implant placement 


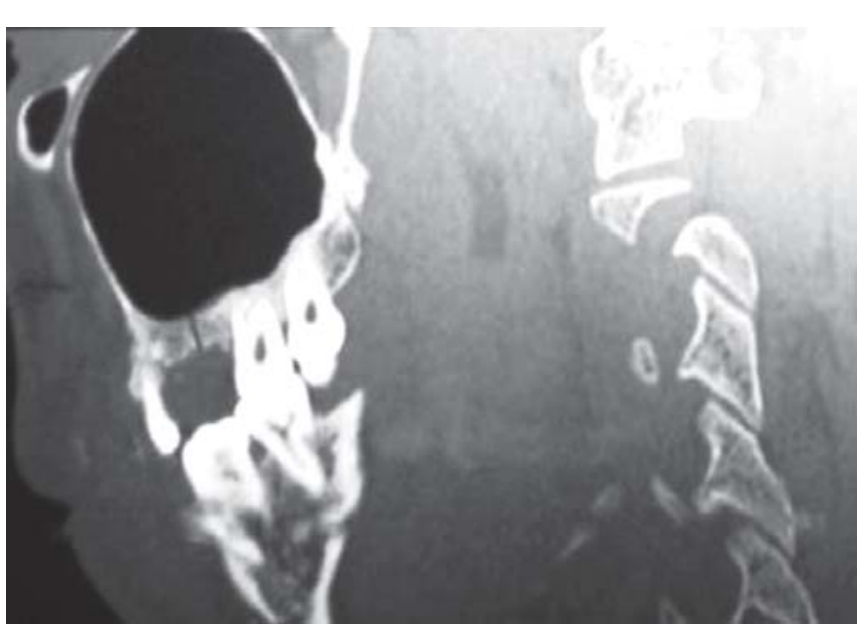

Fig. 2: Dentascan showing available bone height of $5.7 \mathrm{~mm}$ from sinus floor

region was found to be $5.7 \mathrm{~mm}$ from the maxillary sinus lining (Fig. 2). In this case, criteria, such as position of the implant, preexisting tooth form and position, its relation with the opposing arch, soft tissue anatomy, maxillary sinus anatomy and bone dimensions were considered. After thorough oral and radiographic examination, two-stage surgery was planned. It was decided to lift up the sinus lining with an indirect approach through alveolar crest and simultaneously placing the implant. An endopore implant of $7 \mathrm{~mm}$ length and $4.1 \mathrm{~mm}$ diameter was selected.

The treatment was phased out in the following manner:

1. Prior to surgery, a surgical template made up of clear acrylic was used and a hole was made so as to decide the location of implant placement. This hole in the template was used in the surgery for making the pilot drill.

2. A midcrestal, full-thickness incision was performed. Once the flap was reflected (Fig. 3), the indirect approach begins with a pilot bur (Fig. 4) to mark the implant site. A $2 \mathrm{~mm}$ twist drill prepares the osteotomy 1 to $2 \mathrm{~mm}$ short of the sinus floor.

3. Before using the osteotome, a small amount of alloplastic bone graft material was placed into the implant site using amalgam carrier (Fig. 5). This material will act as a shock absorber when tapping of osteotome is done with the mallet, for increasing the diameter of implant site.

4. The implant osteotomy site was then prepared to full dimension by osteotomes of increasing diameter (Fig. 6). The sinus floor was fractured with repeated bone grafting and osteotome insertion.

5. An endopore implant was then tapped gently into the prepared site. This procedure pushed the bone graft material upward, leading to lifting of the sinus membrane $2 \mathrm{~mm}$ ahead of the implant. The primary stability of the implant was checked.

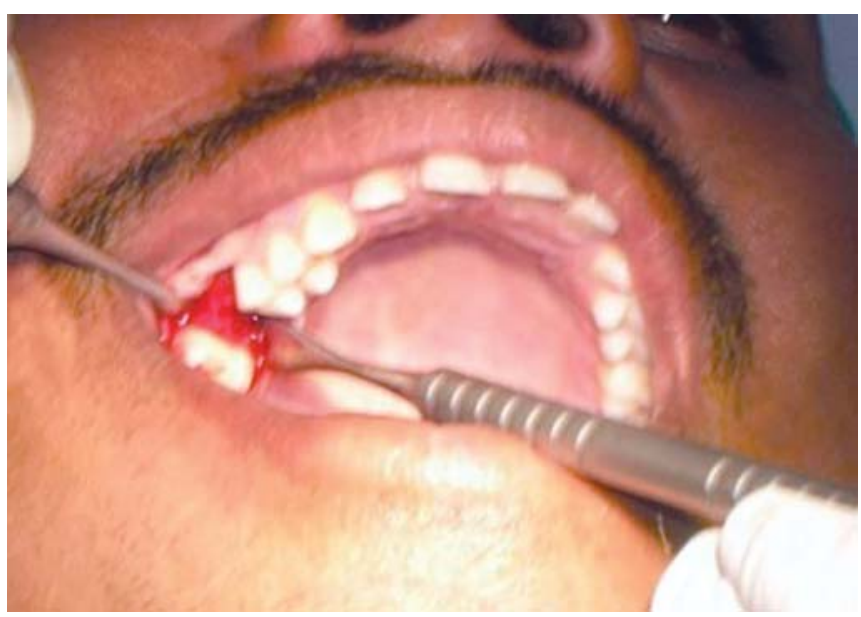

Fig. 3: A midcrestal, full-thickness incision

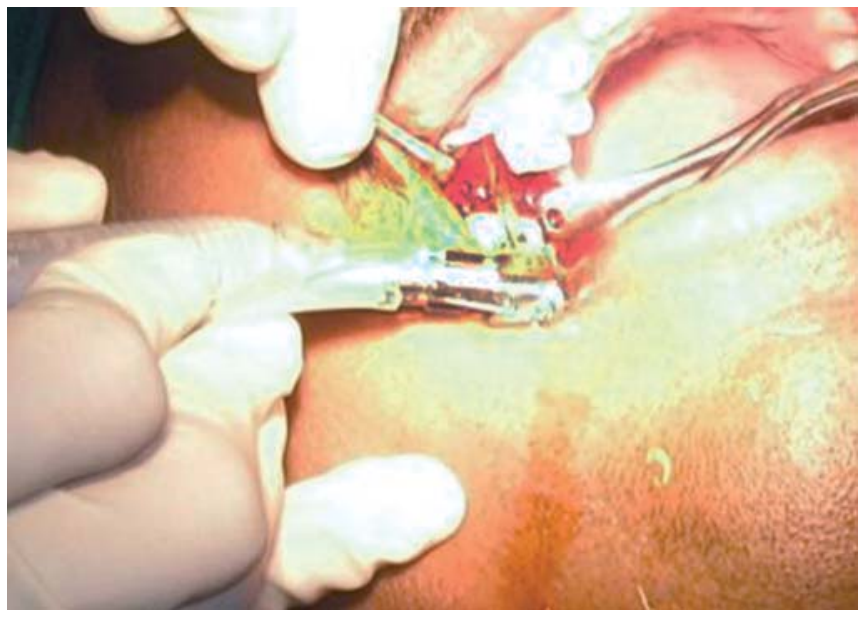

Fig. 4: Pilot drilling for marking the implant site

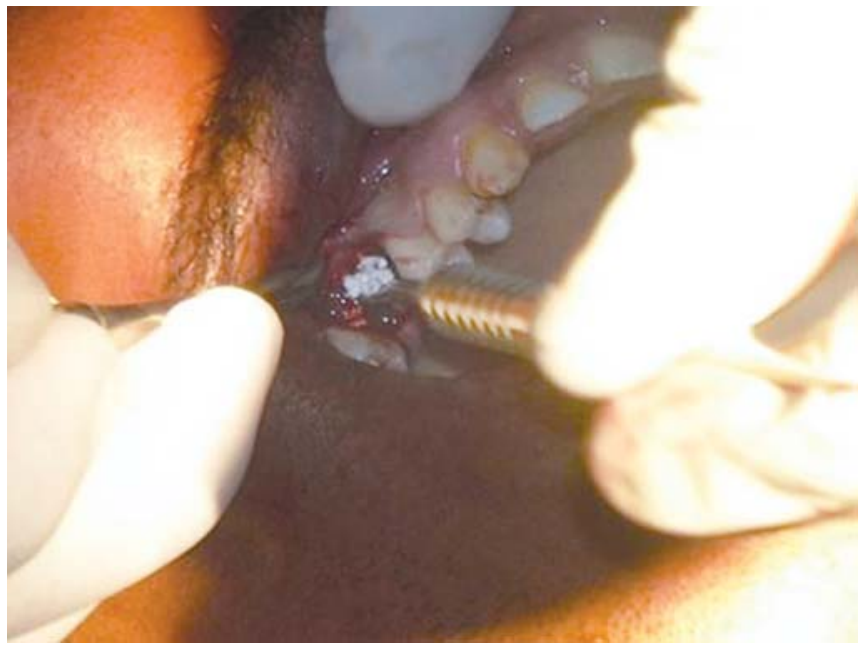

Fig. 5: Placement of bone graft

6. The cover screw was then placed, the implants were covered with the mucosa and sutures were placed.

7. After 7 days, sutures were removed and after 3 months, prior to implant exposure, radiograph was made so as to check for the osseointegration (Fig. 7). After 


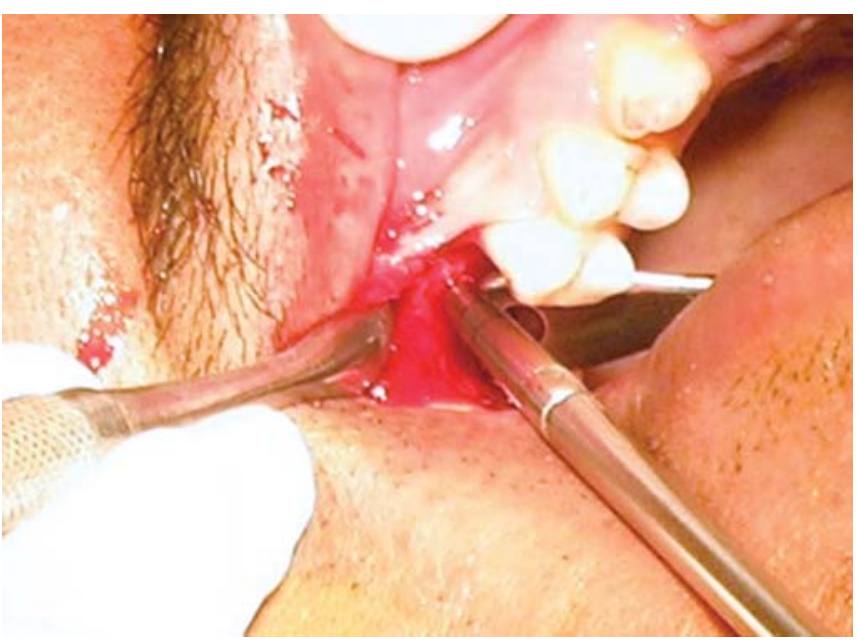

Fig. 6: Osteotome insertion

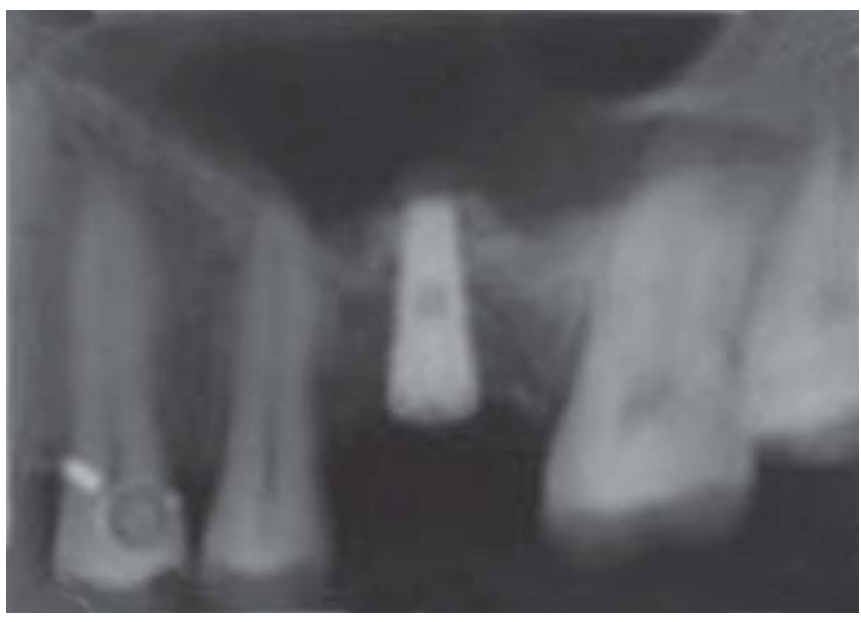

Fig. 7: Intraoral periapical radiograph after 3 months showing osseointegration

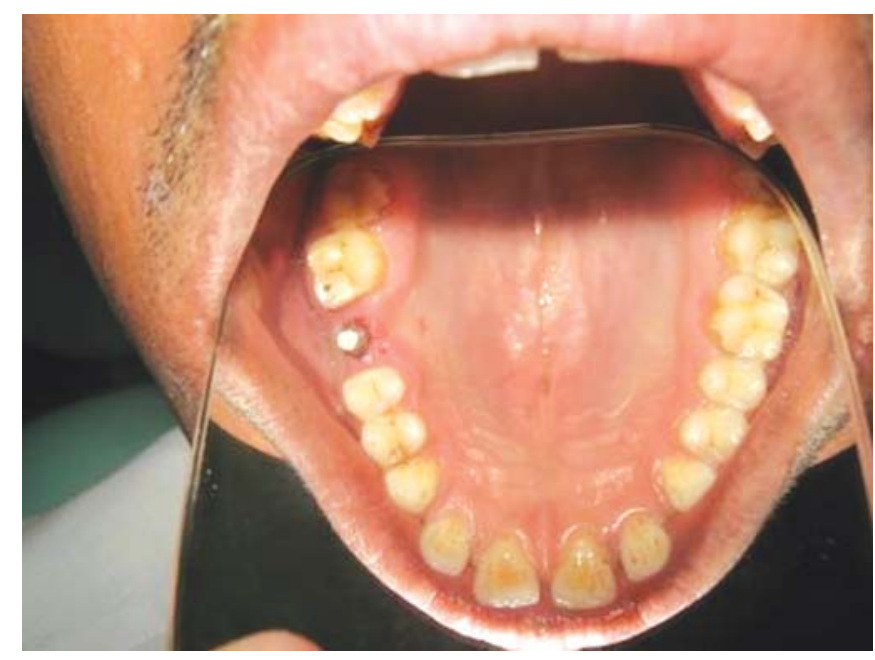

Fig. 8: Abutment in place

satisfactory results, the implant was exposed and covered with the gingival former, so as to get the proper contour of the gingiva.

8. After a week, abutment was chosen (Fig. 8) and with the help of transfer coping, impression was made so as

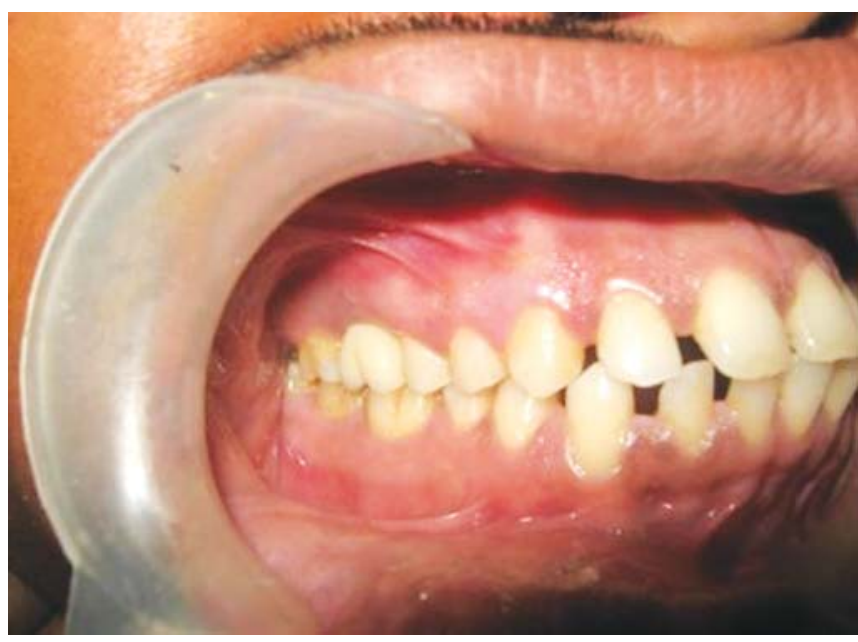

Fig. 9: Postoperative photograph showing final prosthesis

to get the master cast. The wax pattern was fabricated and casted and a metal framework was obtained.

9. The implant supported metal ceramic fixed prosthesis was fabricated (Fig. 9). Patient was instructed about the maintenance of oral hygiene by means of dental floss, interdental brush and mouthwash. Also, the patient was called upon for recall visits after 1 week, 1 and 3 months.

\section{DISCUSSION}

With the advancement in the field of dentistry, implant supported prosthesis are no more a big challenge. When the width, height and quality of alveolar ridge are adequate and satisfactory, the implant placement becomes an easy task but when alveolar ridges are severely resorbed, the bone volume must be increased before implants may be placed. Placement of implants is of more concern in posterior maxilla because of the presence of maxillary sinus. As the ridge resorbs, the sinus lining comes closer to the ridge, leading to chances of sinus perforation during implant placement. In such situations, it is mandatory to lift up the sinus lining and increasing the bone height before implant placement.

Many techniques have been demonstrated by different authors for maxillary sinus lift up. The sinus lift can be achieved by two approaches: (1) Using direct approach and (2) using indirect approach. In direct approach, the sinus is approached from lateral side using one step or two step antrostomy whereas in indirect approach, sinus is approached from crest of the alveolar ridge and an osteotome is used.

This article describes a technique that used indirect approach for lifting up the sinus lining by use of an endopore implant. The sinus floor was first fractured with the use of bone graft and osteotome but the lining was not elevated at this time. Unlike the self-threaded implants, endopore 
implants require tapping and because of this property, they were used for lifting up the sinus membrane.

In this case report, the radiographic examination revealed sinus to be $5.7 \mathrm{~mm}$ from the alveolar ridge. An endopore implant of $7 \mathrm{~mm}$ length and $4.1 \mathrm{~mm}$ diameter was used for elevation of sinus lining. Around $4 \mathrm{~mm}$ of rise in the sinus membrane is done so that after placement of implant, around $2 \mathrm{~mm}$ of graft material remains between the implant and sinus membrane. The advantages of using indirect method over the direct one is that this procedure is less complex, less invasive and have a shorter healing and waiting period.

\section{CONCLUSION}

It can be seen that many advanced surgical techniques exist to overcome the expected anatomical difficulties during implantation. It is important, however, to remember the desired treatment outcome and to explore all the possible solutions. Even in compromised situation, by good evalution of both patient's desires and the available possibilities and by choosing the suitable technique, keeping the technique as simple and as predictable as possible, the likelihood of success increases greatly.

\section{REFERENCES}

1. Small SA, Zinner ID, Panno FV, et al. Augmentation of the maxillary sinus for implants: Report of 27 patients. Int J Oral Maxillofac Implants 1993;8:523.

2. Garg A. Augmentation grafting of the maxillary sinus for the placement of dental implants: Anatomy, physiology and procedure. Implant Dent 1994;8:36.

3. Thomas GJ. Sinus lifts. A possible solution to the atrophic maxilla. J Macomb Dent 1990;29:9.

4. Kraut R, Kesler H. Quantification of bone in dental implant sites after composite grafting of the mandible. Int J Oral Maxillofac Implants 1989;4:143.

5. Smiller DG, Johnson PW, Lozada JL, et al. Sinus lift grafts and endosseous implants. Dent Clin North Am 1992; 36:151.

\section{ABOUT THE AUTHORS}

\section{Amit Agnihotri (Corresponding Author)}

Senior Lecturer, Department of Oral and Maxillofacial Surgery, Guru Gobind Singh College of Dental Science and Research Centre, Lalbagh Road, Burhanpur, Madhya Pradesh, India, Phone: +919039245980 e-mail: shikha_198302@yahoo.co.in

\section{Deepshikha Agnihotri}

Senior Lecturer, Department of Prosthodontics, Guru Gobind Singh College of Dental Science and Research Centre, Burhanpur, Madhya Pradesh, India 